$2-13-2020$

\title{
Temporal Trends in the Standing Broad Jump Performance of United States Children and Adolescents
}

\author{
Bridget K. Pinoniemi \\ Grant R. Tomkinson \\ University of North Dakota, grant.tomkinson@und.edu \\ Tanis J. Walch \\ University of North Dakota, tanis.walch@und.edu \\ James N. Roemmich \\ John S. Fitzgerald \\ University of North Dakota, john.s.fitzgerald@UND.edu
}

\section{How does access to this work benefit you? Let us know!}

Follow this and additional works at: https://commons.und.edu/ehb-fac

Part of the Exercise Science Commons

\section{Recommended Citation}

Bridget K. Pinoniemi, Grant R. Tomkinson, Tanis J. Walch, et al.. "Temporal Trends in the Standing Broad Jump Performance of United States Children and Adolescents" (2020). Education, Health \& Behavior Studies Faculty Publications. 52.

https://commons.und.edu/ehb-fac/52

This Article is brought to you for free and open access by the Department of Education, Health \& Behavior Studies at UND Scholarly Commons. It has been accepted for inclusion in Education, Health \& Behavior Studies Faculty Publications by an authorized administrator of UND Scholarly Commons. For more information, please contact und.commons@library.und.edu. 


\section{Title}

Temporal Trends in the Standing Broad Jump Performance of United States Children and Adolescents 


\begin{abstract}
Purpose: To estimate temporal trends in broad jump performance for United States youth, a marker of muscular fitness and health.

Method: Electronic databases, topical systematic reviews, and personal libraries were systematically searched for studies reporting descriptive standing broad jump data for apparently healthy United States youth (age 10-17 years). Temporal trends at the sex-age level were estimated using sample-weighted regression models associating the year of testing to mean jump performance, with national trends standardized to the year 1985 using a post-stratified population-weighting procedure.

Results: Collectively, there was a small increase of $12.6 \mathrm{~cm}(95 \% \mathrm{CI}: 12.5$ to 12.7$)$ or $7.9 \%$ (95\%CI: 7.1 to 8.6) in 65,527 United States youth between 1911 and 1990. Increases were greater for girls (change in means [95\% CI]: $17.1 \mathrm{~cm}$ [16.9 to 17.3$] ; 11.4 \%$ [10.7 to 12.2$]$ ) compared to boys (change in means [95\% CI]: $8.5 \mathrm{~cm}$ [8.3 to 8.7]; 4.6\% [3.8 to 5.4]), but did not differ between children (10-12 years) and adolescents (13-17 years). Increases in broad jump performance were not always uniform across time, with steady and progressive increases observed for boys and children, respectively, and a diminishing rate of increase observed for girls and adolescents.
\end{abstract}

Conclusions: Muscular fitness is a good marker of health, so greater broad jump performance from 1911 to 1990 may reflect corresponding changes in health. Routine assessment of broad jump performance may be useful to monitor trends in health and muscular fitness of United States youth due to its practicality, scalability, and predictive utility.

Keywords: secular changes; physical fitness; muscle strength; youth. 


\section{Introduction}

Physical fitness is an important marker of general health (Ortega, Ruiz, Castillo, \& Sjöström, 2008; Ross et al., 2016). Although cardiorespiratory fitness has long been considered a powerful marker of health, the importance of muscular fitness (MF) to health has recently emerged, with the majority of the literature available for adults. In adults, lower MF is associated with mortality (all-cause, cardiovascular and non-cardiovascular) (Leong et al., 2015), stroke ( $\mathrm{berg}$ et al., 2015), diabetes (Mainous, Tanner, Anton, \& Jo, 2015), disability (Phillips \& Haskell, 1995), falls (Chan et al., 2007), cognitive declines (Alfaro-Acha et al., 2006), and functional deficits (McGrath, Robinson-Lane, Peterson, Bailey, \& Vincent, 2018). In youth, MF is associated with both current and future health. In a meta-analysis, Smith and colleagues (2014) reported that lower youth MF was associated with lower self-esteem, poorer bone health, higher body fat, and greater cardiometabolic risk. Longitudinal evidence suggests youth MF is also associated with reduced adiposity, reduced cardiometabolic risk, and improved bone health in later life (GarcíaHermoso, Ramírez-Campillo, \& Izquierdo, 2019). In a cohort of over one million males with a median follow-up of 24 years, Ortega and colleagues (2012) found that greater MF in adolescence was associated with a $20-35 \%$ risk reduction in cardiovascular, suicide, and allcause mortality, with adolescents falling below the tenth percentile for MF being at the greatest risk for all-cause mortality.

MF has multiple components (strength, power/explosive strength, and endurance) and its assessment is a task-specific evaluation of the motor system (neural and muscle function). MF has been evaluated using several measures and the National Academy of Medicine (formerly known as the Institute of Medicine) (Institute of Medicine, 2012), as well as European experts 
(Ruiz et al., 2011), has recommended the handgrip strength and broad jump for school-based fitness testing because of their health-related predictive utility. Jumping performance - a measure of lower-body power/explosive strength — has been meaningfully associated with health and consistently correlated with vigorous physical activity levels in youth (Smith et al., 2019). The consistent relationship between jumping performance and physical activity is likely due to correspondence: jumping, leaping, and running are natural movements commonly performed by youth during leisure time activity and physical education. In addition, jumping tests are highly reliable (Docherty, 1996), moderately-to-highly valid (Castro-Piñero et al., 2010; Milliken, Faigenbaum, Loud, \& Westcott, 2008), and scalable for school-based fitness testing (Institute of Medicine, 2012). Thus, jumping performance seems a good fit for tracking temporal trends in youth MF and for determining the efficacy of public health efforts to improve youth MF.

Temporal trends in MF of United States youth have largely focused on handgrip strength, with trends spanning 110 years over the period 1899-2009 (Espenschade \& Meleney, 1961; Hunsicker \& Reiff, 1977; Malina, 1978; Montpetit, Montoye, \& Laeding, 1967; Reiff et al., 1986; Silverman, 2011, 2015). While much less is known about trends in broad jump performance of United States youth, the most comprehensive analysis to date suggests that broad jumping performance improved over the last part of the $20^{\text {th }}$ century (Tomkinson, 2007). In a systematic analysis of 20.8 million youth (aged 6-19 years) from 23 countries, Tomkinson (2007) indicated that broad jump performance improved at $2.6 \%$ per decade between 1970 and 1989 in 39,937 North American youth. However, it is difficult to determine how well these findings reflect temporal trends in the broad jump performance of United States youth because 
Canadian and United States data were combined in Tomkinson (2007) to estimate the regional trend for North America. Population-specific broad jump performance temporal trends may provide better insight into trends for United States youth. The current study, restricted to United States youth, provides an update of our previous study (Tomkinson, 2007) by extending the temporal picture and updating the data pooling methods. Using a systematic review approach, the aim of this study was to estimate the temporal trends in broad jump performance for United States youth. It was hypothesized that broad jump performance had improved over time at a magnitude similar to that reported by Tomkinson (2007).

\section{Methods}

\section{Data Sources}

An electronic database search was performed on the $27^{\text {th }}$ of November 2018 , using SPORTDiscus, CINAHL, and MEDLINE without date or language restrictions. The search strategy was developed in consultation with an academic librarian experienced in systematic review searching. Additional studies were located by searching reference lists, topical systematic reviews, and the personal library of one of the authors. The protocol for this systematic review was registered with the International Prospective Register of Systematic Reviews (PROSPERO; registration number: CRD42019125072) and it followed the Preferred Reporting Items for Systematic reviews and Meta-Analyses statement (Moher, Liberati, Tetzlaff, \& Altman, 2009).

\section{Inclusion and Exclusion Criteria}

Studies reporting descriptive data (sample sizes, means, and/or standard deviations) for apparently healthy (no known disease, signs/symptoms of disease, or injury/illness) United 
States youth (age 10-17 years) tested on the broad jump tests were included. Descriptive data must have been reported at the age-sex-year level (e.g., 10-year-old boys tested in 1975). Broad jump performance was operationalized as a standing horizontal double-legged jump for the greatest possible distance.

\section{Search Strategy}

The electronic database search was restricted to keywords, abstract, and title. The Boolean OR was used to combine terms within a group, and the Boolean AND was used to combine search groups. Proximity operators were used to search for root words. Three search groups were used: the first described the fitness measure ("musc* fitness" OR "musc* strength" OR "musc* power" OR "jump*"); the second described the population ("child*” OR "adolescen*” OR "boy*" OR "girl*" OR "youth"); and the third described the geographical location ("America*" OR “U.S.*”).

\section{Study Selection}

Database records were imported into RefWorks (v2.0; ProQuest LLC, Ann Arbor, MI, USA) and de-duplicated. At the first level, two researchers independently screened the titles and abstracts against inclusion criteria, with consensus required for further screening. At the second level, full text copies were obtained and independently screened by two researchers against inclusion criteria, with consensus required for final inclusion. A third researcher resolved discrepancies if consensus was not reached.

\section{Data Extraction}


Descriptive data were extracted into Excel (Microsoft Corp., Redmond, WA, USA) using a predetermined template (Tomkinson, Lang, \& Tremblay, 2019) and reviewed by a second researcher for accuracy. Email contact was made with corresponding authors if additional information was required (e.g., to clarify published results).

The following study-specific data were extracted: author, sampling frame (national, state/regional, or other [city/district/school]), sampling method (probability or non-probability), year of testing, sex, age (calendar age), broad jump test protocol, sample size, mean, standard deviation, and/or median. All mean, standard deviation, and median data were converted to the common metric of centimeters. Testing year was recorded as the midpoint year of testing, with two years prior to the publication year assumed for studies when missing, as this was the median difference for those studies in which the testing year was known.

Missing means were estimated from the reported medians using the equation: mean $=0.978 \times$ median +3.209 , where $r=0.997$ and $\mathrm{SEE}=1.7 \mathrm{~cm}$. This equation was generated by first locating all studies reporting both medians and means at the sex-age-year level and second, by determining the best-fitting and most parsimonious linear or curvilinear (quadratic or cubic polynomials) regression models between median (predictor variable) and mean (response variable) values. Missing standard deviations were estimated from sample-weighted mean coefficient of variation of $15.2 \%$. This sample-weighted mean coefficient of variation was estimated by first locating all studies reporting both means and standard deviations at the sexage-year level; second, by calculating the corresponding sex-age-year coefficients of variation; and third, by calculating the sample-weighted mean coefficient of variation. 


\section{Data Synthesis}

Temporal trends were analyzed at the sex-age level using sample-weighted linear or polynomial (quadratic or cubic) regression models relating the year of testing to mean broad jump performance (Lamoureux et al., 2019; Tomkinson et al., 2019). Trends in mean broad jump performance were expressed as absolute changes in means, percentage changes (i.e., change in means expressed as a percentage of the overall mean), and as standardized effect sizes (ES) (i.e., change in means divided by the pooled standard deviation). ES of $0.2,0.5$, and 0.8 were used as thresholds for small, moderate, and large, respectively, with $\mathrm{ES}<0.2$ considered to be negligible (Cohen, 1988). Positive temporal trends indicated increases in mean broad jump performance and negative temporal trends indicated declines in mean broad jump performance.

National trends (for boys, girls, children [10-12 year-olds], adolescents [13-17 year-olds], and all [10-17 year-olds]) were calculated using a post-stratified population-weighting procedure that has been described in detail elsewhere (Lamoureux et al., 2019; Tomkinson et al., 2019). Population estimates were standardized to the year 1985-a common testing year to all sex-age groups - using United Nations (2019) data. The post-stratification population-weighting procedure helped to correct the national trends for sampling bias by standardizing the trends to underlying country-sex-age-specific demographics. National trends were also examined for nonuniformity by: (a) using the $\mathrm{D}_{\max }$ method (Cheng et al., 1992) to locate a breakpoint; (b) fitting linear regression models to data points before and after the breakpoint; and (c) comparing the resultant linear trends. 


\section{Results}

Study Selection

A total of 828 unique records were obtained from the electronic database search, with 23 retained after the first level of screening (title and abstract review) and 1 retained after the second level of screening (full-text review) (Figure 1). An additional 11 studies were identified by searching reference lists, topical systematic reviews, and personal libraries, resulting in a total of 12 studies (Bos, 1961; Brogdon, 1972; Corroll, 1967; Espenschade \& Meleney, 1961; Fleishman, 1964; Hunsicker \& Reiff, 1977; Ikeda, 1962; Katzmarzyk, Malina, \& Beunen, 1997; Koebel, Swank, \& Shelburne, 1992; Reiff et al., 1986; Robbins, 1984; Weber, 1969) representing 16 unique datasets.

***Figure 1 here $* * *$

\section{Study Characteristics}

Broad jump data were available for 62,527 United States youth (34,569 boys and 27,957 girls) aged 10-17 years between 1911 and 1990 (Table 1). Data were available for 180 sex-age-year groups, with an average sample size of 347 per group. The majority of data sets were obtained using non-probability sampling (68\%) and were not national or state/regional samples (53\%).

$$
* * * \text { Table } 1 \text { here } * * *
$$

\section{Overall Trend}

Collectively, there was a small improvement in mean broad jump performance between 1911 and 1990 (change in means [95\% CI]: $12.6 \mathrm{~cm}[12.5$ to 12.7$] ; 7.9 \%$ [7.1 to 8.7$] ; 0.49$ ES [0.46 to 0.53]) (Figure 2). Figure 2 (bottom panel) suggests that the national rate of improvement slowed in the latter decades. This was confirmed by $\mathrm{D}_{\max }$ analysis which located a breakpoint at 1965 , 
with rates of improvement pre-1965 (change in means [95\% CI]: 1.1\% per decade [1.0 to 1.2]) nearly double those post-1965 (change in means [95\% CI]: $0.6 \%$ per decade [0.5 to 0.7$]$ ).

\section{Trends in Children and Adolescents}

There was a negligible difference in the trends between children and adolescents, with a moderate improvement in children (change in means [95\% CI]: $11.8 \mathrm{~cm}$ [11.6 to 12.0$] ; 8.2 \%$ [7.4 to 9.0$] ; 0.52$ ES [0.44 to 0.59$]$ ) and a small improvement in adolescents (change in means [95\% CI]: $13.4 \mathrm{~cm}$ [13.2 to 13.6]; $7.8 \%$ [7.1 to 8.6]; 0.49 ES [0.41 to 0.57]) (Figure 2). Despite similar magnitudes of improvement, Figure 2 (middle panels) suggests an increasing rate of improvement in children over the entire period and a decreasing rate of improvement in adolescents. $D_{\max }$ analysis located breakpoints at 1950 and 1959 for children and adolescents, respectively, with rates of improvement increasing 2-fold after 1950 in children (change in means [ $95 \% \mathrm{CI}]$ : pre-1950, $0.7 \%$ per decade [0.5 to 0.9$]$; post- $1950,1.4 \%$ per decade [ 1.3 to 1.5]) and decreasing 4-fold after 1959 in adolescents (change in means [95\% CI]: pre-1959, $1.3 \%$ per decade [1.2 to 1.4$]$; post- $1959,0.3 \%$ per decade [0.1 to 0.5$]$ ).

\section{Trends in Boys and Girls}

There was a small-to-moderate difference in the trends between boys and girls, with a moderate improvement in girls (change in means [95\% CI]: $17.1 \mathrm{~cm}$ [16.9 to 17.3$]$; $11.4 \%$ [10.7 to 12.2$]$; $0.72 \mathrm{ES}$ [0.64 to 0.79]) and a small improvement in boys (change in means [95\% CI]: $8.5 \mathrm{~cm}$ [8.3 to 8.7 ]; $4.6 \%$ [3.8 to 5.4]; 0.29 ES [0.21 to 0.37]) (Figure 2). Despite a 2.5 -fold difference in the percent magnitude of improvement, Figure 2 (top panels) suggests a steady improvement over time in boys and a slowing of the rate of improvement in girls. $\mathrm{D}_{\max }$ analysis located 
breakpoints at 1968 and 1963 for boys and girls respectively, with no difference in rates of improvement pre- and post-1968 in boys (change in means [95\% CI]: pre-1968, 0.6\% per decade [0.5 to 0.7 ]; post-1968, $0.4 \%$ per decade [0.2 to 0.6$]$ ), yet a 2 -fold decrease after 1963 in girls (change in means [95\% CI]: pre-1963, 1.6\% per decade [1.5 to 1.7]; post-1963, 0.8\% per decade [0.6 to 1.0$])$.

***Figure 2 here***

\section{Discussion}

This systematic analysis examined temporal trends in the broad jump performance of United States youth over the 79-year period from 1911 to 1990 and found: (a) a small improvement in broad jump performance; (b) similar improvements in children and adolescents; (c) a 2.5-fold greater improvement in girls compared to boys; and (d) generally curvilinear temporal improvements. The absence of broad jump data after 1990, combined with its health-related predictive utility, underscore the potential importance for fitness surveillance systems to reintroduce the broad jump test to assess the current status of, and trends in, youth MF in the United States. These data could be used to inform policy-making and public health planning.

\section{Comparisons with Other Studies}

While numerous studies have recently reported on temporal trends in youth broad jump performance (Australia [Fraser et al., 2018]; Greece [Smpokos, Linardakis, Papadaki, Lionis, \& Kafatos, 2012]; Lithuania [Venckunas, Emeljanovas, Mieziene, \& Volbekiene, 2017]; New Zealand [Albon, Hamlin, \& Ross, 2010]; Poland [Ignasiak, Slawińska, \& Malina, 2016]; Spain [Moliner-Urdiales et al., 2010]; and the UK [Sandercock \& Cohen, 2019]) the most comprehensive study to date was a large systematic analysis of over 20 million youth from 23 
countries (Tomkinson, 2007). Tomkinson's systematic analysis indicated a very small international improvement of $0.3 \%$ per decade between 1958 and 2003, although the trend was not uniform over time, with performances improving from the late 1950s to the mid-1980s and declining thereafter. The trend for North American youth was considerably larger than the international trend - 2.6\% vs. 0.3\% improvement per decade, respectively. By way of comparison, between 1958 and 1990, the present study observed a collective improvement of $0.7 \%$ per decade. The temporal difference between the present study and that of the North American youth in Tomkinson (2007) could be because: (a) the North American trend in Tomkinson (2007) was generated by combining trend data for both Canadian and United States youth aged 6-17 years whereas this study only reported trends for United States youth aged 1017 years; and (b) Tomkinson (2007) included only studies that explicitly reported on temporal trends in jump performance whereas this study included studies that reported on broad jump performance even if measured on a single occasion. Lastly, this study expanded the temporal picture of broad jump performance for United States children and youth, albeit backwards and not forwards, which was surprising given the updated data pooling methods and that the broad jump is widely used for school-based MF testing (Tomkinson et al., 2018).

\section{Trends in Maturation}

Advances in maturation likely contributed to the increased MF observed in this study. Changes in body size, shape, and composition occur throughout maturation (Rogol, Clark, \& Roemmich, 2000) and modify MF performance. It appears the timing of this process, which is influenced by genetic, endocrine, and environmental factors such as nutrition (Özen \& Darcan, 2011) has shifted to an earlier chronological age. Data suggest that maturation is advancing at a rate of 
about 2 and 3 months per decade for boys (the age boys' voices break) and girls (age of menarche), respectively (Roche, 1979). Thus, a 13-year-old boy in 1911 would be maturationally similar to a 12-year-old in 1990 and a 13-year-old girl would be maturationally similar to an 11year-old in 1990. Reiff et al. (1986) found that broad jump performance improved with each year of age by $5.7 \%$ in boys and $2.2 \%$ in girls between the ages of 10 and 17 . Over the 79 -year period, improvements of $9.0 \%$ for boys (i.e., 7.9 [decades] $\times 0.2$ [years per decade] $\times 5.7 \%$ [age-related change]) and $5.2 \%$ for girls (i.e., 7.9 [decades] $\times 0.3$ [years per decade] $\times 2.2 \%$ [age-related change]) would be expected from maturational advances alone. Corresponding improvements in broad jump performance were $4.6 \%$ and $11.4 \%$ for boys and girls, respectively. While only an estimate, these data suggest that for girls, advances in maturation explain a meaningful proportion of the observed improvement in broad jump performance; for boys, they suggest that the observed improvement might reflect an underlying decline. Since calendar age was used to estimate temporal trends in this study, the divergence of chronological and biological age likely contributed to improved MF (because MF would generally favor youth of the same chronological age in the more recent surveys), and may help explain the sex- and age-related temporal differences.

Body size, which includes height and mass, has also increased over time (Karpati, Rubin, Kieszak, Marcus, \& Troiano, 2002). On average, height has increased approximately 1.5 and 2.5 centimeters per decade for children and adolescents respectively, between 1880 and 1980 in Europe and North America (Hauspie, Vercauteren, \& Susanne, 1997). The long bones, which consist of the arms and the legs, are known to grow first during a pubertal growth spurt (Cole, 2000). Although not population-specific, evidence suggests increased leg length as the primary 
contributor to observed height increases (Malina, 2004). Given the nature of broad jump performance, increased height and associated leg length may afford an advantage by increasing takeoff and landing horizontal displacement, independent of displacement during the flight phase. This mechanical advantage may have contributed to the trends of increased MF over time.

Until the latter years, youth body mass, relative to height, appeared to have remained fairly stable. Using data from the National Health and Nutrition Examination Survey of United States

youth, Ogden, Flegal, Carroll, and Johnson (2002) reported the prevalence of overweight to have increased by $7 \%$ in $6-11$ year olds and by $6 \%$ in $12-19$ year olds from the 1960 s to $1988-94$. However, the majority of the increase in the prevalence of overweight occurred after 1976-80 (Malina, 2004; Ogden et al., 2002). It is possible the additional adiposity associated with overweight status may have contributed to slowing the rate of improvement in this study. It appears compensatory gains in muscle mass, associated with increased weight status, offset the negative influence of added adiposity on jump performance, at least at the levels specific to the study period. Although, a threshold likely exists at the population level where increased BMI negatively influences jumping performance. The plateauing of adolescents' broad jump performance near 1980 (Figure 2) may be evidence of such and is consistent with studies around the globe reporting recent increases in BMI and declines in jumping performance post-1980 (Albon et al., 2010; Fraser et al., 2018).

\section{Trends in Social/Public Health Factors}

Temporal trends in broad social, environmental, and behavioral factors likely contributed to the trends in broad jump performance. Temporal trends of biological maturation and body size are 
thought to be influenced by improved living conditions, better nutrition, reduced disease, and mortality (Malina, 2004). Numerous public health initiatives (e.g., health promotion campaigns, clean water technologies, food fortification, pasteurization) in the United States have contributed to improved environmental and nutritional conditions of youth during the study period (Backstrand, 2002; Cutler \& Miller, 2005), especially during the earlier years, although it is difficult to estimate the magnitude and temporal impact of such factors on MF. Other social (e.g., gender) and psychosocial factors (e.g., intrinsic and extrinsic motives) are meaningfully associated with youth physical activity and sports participation (Weinberg et al., 2000) behaviors which are associated with enhanced MF, particularly vigorous physical activity (Smith et al., 2019). No objectively measured temporal trend data exist indicating changes in overall physical activity levels in youth are available over the study period; however, active transportation (i.e., walking or cycling) has declined by 9\% per decade between 1969 to 2001 in United States youth aged 5-18 years (Booth, Rowlands, \& Dollman, 2015). Although the benefit of cycling to MF in youth is unclear, it likely imposes physical demands that may have improved MF over time (Smith et al., 2019). Declines in active transportation, combined with other broad social and environmental factors (e.g., increased economic growth, changes in the built environment and social climate), may also be representative of a physical activity transition (Katzmarzyk \& Mason, 2009) in the United States resulting in a gradual shift away from moderate and vigorous physical activity. Thus, reductions in active transportation and vigorous physical activity may be involved in the slowing of the rate of improvement from the mid-to-late 1960s in some sex and age groups (e.g., girls and adolescents). While boys' high school sports participation remained relatively stable, girls' participation experienced an abrupt and sustained increase after the enactment of Title IX in 1972 (Bassett, John, Conger, Fitzhugh, \& Coe, 2015), 
which likely contributed to the 2-fold larger improvement in MF in girls compared to boys. Lastly, a reduced national emphasis on the then characterized "performance-related" physical fitness testing in schools after 1965 (Koebel et al., 1992; Malina, 2004), may have impacted physical education curriculum, reducing the time spent performing activities that have the largest impact on lower-body MF, and may help explain the overall slowing rate of improvement after 1965.

\section{Implications}

Emerging evidence indicates that MF is, indeed, meaningfully related to youth health (GarcíaHermoso et al., 2019; Ortega et al., 2012; Smith et al., 2014), highlighting the need for practical, feasible, and scalable measures of MF. Jumping is a fundamental movement skill employed and developed by youth during everyday activities, including leisure time activity, physical education, and sport, and it consistently correlates with youth vigorous physical activity levels (Smith et al., 2019). Considering correspondence to youth physical activity, the broad jump may be better suited to assess changes in MF, compared to other MF tests (e.g., curl-up, push-up, bent arm hang), brought on by school-based or population-based public health interventions. The broad jump could be easily added, as a complement to existing MF tests, to fitness surveillance systems in the United States (e.g., The Presidential Youth Fitness Program [President's Council on Sports, Fitness \& Nutrition, 2018], FITNESSGRAM® [Welk, Bai, Saint-Maurice, AllumsFeatherston, \& Candelaria, 2016]) as the test requires minimal participant, equipment, and administration burden. The broad jump is widely used across the globe (Tomkinson, 2007) and cardiometabolic health-related criterion cut-points have been established in youth (Castro-Piñero et al., 2019). Broad jump performance appears to be declining elsewhere after 1990 (Albon et al., 
2010; Fraser et al., 2018; Moliner-Urdiales et al., 2010; Sandercock \& Cohen, 2019; Tomkinson, 2007) and it has likely trended similarly in United States youth, though population-specific data are required to confirm this trend. Adding the broad jump to existing surveillance systems in the United States would allow for within-country and between-country comparisons that may enhance the ability to identify broad sociodemographic factors related to MF, to develop strategies to address disparities, and to assess the efficacy of population-based interventions.

\section{Strengths and Limitations}

This study used a systematic review approach and a strict set of inclusion/exclusion criteria to locate studies reporting on the broad jump performance of apparently healthy United States youth. It used a statistical approach previously adopted in other systematic review on trends in children's fitness (Tomkinson, 2007; Tomkinson et al., 2019), including weighted regression and post-stratification population weighting to adjust for sampling bias and underlying demographics. However, calculating trends using descriptive data meant that the influence of concurrent trends in body size and maturation (or other mechanistic factors, e.g., trends in physical activity levels) could not be statistically removed. An inclusive strategy was adopted where data collected on national, state/regional, and community samples were included, which raises the issue of representativeness. Only $21 \%$ (4/19) of data sets were national surveys utilizing probability sampling. However, given the first national fitness survey was conducted in the late 1950s, the inclusion of state/regional and community level data, which represent the best available data on United States youth at the time, allowed for a more complete temporal picture. Trends in ethnic mix of the US population may have biased our trends in broad jump, although we could not estimate the impact of such trends because sample-specific ethnicity data were not 
always reported. It is also possible that assessment procedures (e.g., number of trials, level of encouragement, jumping surface, diurnal variation) varied over time as detailed protocol information was not always available. However, the broad jump was always performed as a double-leg countermovement jump and reported as either the better of two or best of three trials. Differences in protocols among studies were likely small and unlikely to explain our trends in broad jump performance.

\section{Conclusion}

There has been a small improvement in the broad jump performance of United States youth between 1911 and 1990. The broad jump test is no longer used as part of routine fitness surveillance systems extant in the United States, despite the National Academy of Medicine's recommendation for its inclusion in school-based fitness testing. The inclusion of the broad jump, or other jumping tests, in future fitness surveys of United States youth may be useful to monitor trends in health and fitness and to inform healthy public policy.

\section{What Does This Article Add?}

MF is an important marker of current and future health. Temporal trends in the MF of United States youth have largely focused on handgrip strength, and little is known about trends in broad jump performance. This study provides an update of a previous global review, restricted to United States youth. Fitness surveillance systems might consider reinstating the broad jump test as a complementary MF measure to assess recent trends in United States youth. Trends in youth MF may reflect trends in youth health and can potentially inform public health policy. 


\section{References}

Åberg, N. D., Kuhn, H. G., Nyberg, J., Waern Margda, Friberg Peter, Svensson Johan, ... Nilsson Michael. (2015). Influence of Cardiovascular Fitness and Muscle Strength in Early Adulthood on Long-Term Risk of Stroke in Swedish Men. Stroke, 46(7), 17691776. https://doi.org/10.1161/STROKEAHA.115.009008

Albon, H. M., Hamlin, M. J., \& Ross, J. J. (2010). Secular trends and distributional changes in health and fitness performance variables of 10-14-year-old children in New Zealand between 1991 and 2003. British Journal of Sports Medicine, 44(4), 263-269. https://doi.org/10.1136/bjsm.2008.047142

Alfaro-Acha, A., Snih, S. A., Raji, M. A., Kuo, Y.-F., Markides, K. S., \& Ottenbacher, K. J. (2006). Handgrip Strength and Cognitive Decline in Older Mexican Americans. The Journals of Gerontology: Series A, 61(8), 859-865. https://doi.org/10.1093/gerona/61.8.859

Backstrand, J. R. (2002). The history and future of food fortification in the United States: A public health perspective. Nutrition Reviews, 60(1), 15-26. https://doi.org/10.1301/002966402760240390

Bassett, D. R., John, D., Conger, S. A., Fitzhugh, E. C., \& Coe, D. P. (2015). Trends in Physical Activity and Sedentary Behaviors of United States Youth. Journal of Physical Activity \& Health, 12(8), 1102-1111. https://doi.org/10.1123/jpah.2014-0050

Booth, V. M., Rowlands, A. V., \& Dollman, J. (2015). Physical activity temporal trends among children and adolescents. Journal of Science and Medicine in Sport, 18(4), 418-425. https://doi.org/10.1016/j.jsams.2014.06.002 
Bos, R. (1961). An Analysis of the Youth Fitness Project Data and a Comparison of These Data with Comparable Data Recorded Prior to 1940. (Unpublished Doctoral Dissertation). University of Michigan, Ann Arbor, United States.

Brogdon, G. (1972). A Comparison of Physical Fitness and Anthropometric Measures of PreAdolescent Mexican-American and Anglo-American Males (Doctoral Dissertation). North Texas State University, Denton, United States.

Castro-Piñero, J., Artero, E. G., España-Romero, V., Ortega, F. B., Sjöström, M., Suni, J., \& Ruiz, J. R. (2010). Criterion-related validity of field-based fitness tests in youth: A systematic review. British Journal of Sports Medicine, 44(13), 934-943. https://doi.org/10.1136/bjsm.2009.058321

Castro-Piñero, J., Perez-Bey, A., Cuenca-Garcia, M., Cabanas-Sanchez, V., Gómez-Martínez, S., Veiga, O. L., ... UP\&DOWN Study Group. (2019). Muscle Fitness Cut Points for Early Assessment of Cardiovascular Risk in Children and Adolescents. The Journal of Pediatrics, 206, 134-141.e3. https://doi.org/10.1016/j.jpeds.2018.10.026

Chan, B. K. S., Marshall, L. M., Winters, K. M., Faulkner, K. A., Schwartz, A. V., \& Orwoll, E. S. (2007). Incident Fall Risk and Physical Activity and Physical Performance among Older MenThe Osteoporotic Fractures in Men Study. American Journal of Epidemiology, 165(6), 696-703. https://doi.org/10.1093/aje/kwk050

Cheng, B., Kuipers, H., Snyder, A. C., Keizer, H. A., Jeukendrup, A., \& Hesselink, M. (1992). A New Approach for the Determination of Ventilatory and Lactate Thresholds. International Journal of Sports Medicine, 13(07), 518-522. https://doi.org/10.1055/s2007-1021309 
Cohen, J. (1988). Statistical Power Analysis for the Behavioral Sciences $\left(2^{\text {nd }}\right.$ ed). Hillsdale, NJ: Lawrence Erlbaum Associates, Publishers.

Cole, T. J. (2000). Secular trends in growth. The Proceedings of the Nutrition Society, 59(2), 317-324. https://doi.org/10.1017/s0029665100000355

Corroll, V. (1967). AAHPER Youth Fitness Test Items and Maximal Oxygen Intake (Doctoral Dissertation). University of Illinois, Urbana-Champaign, United States.

Cutler, D., \& Miller, G. (2005). The role of public health improvements in health advances: The twentieth-century United States. Demography, 42(1), 1-22. https://doi.org/10.1353/dem.2005.0002

Docherty, D. (1996). Field Tests and Test Batteries. In Measurement in pediatric exercise science (pp. 285-943). Champaign, IL: Human Kinetics.

Espenschade, A. S., \& Meleney, H. E. (1961). Motor Performances of Adolescent Boys and Girls of Today in Comparison with Those of 24 Years Ago. Research Quarterly. American Association for Health, Physical Education and Recreation, 32(2), 186-189. https://doi.org/10.1080/10671188.1961.10613132

Fleishman, E. A. (1964). The structure and measurement of physical fitness. Oxford, England: Prentice-Hall.

Fraser, B. J., Blizzard, L., Schmidt, M. D., Juonala, M., Dwyer, T., Venn, A. J., \& Magnussen, C. G. (2018). Childhood cardiorespiratory fitness, muscular fitness and adult measures of glucose homeostasis. Journal of Science and Medicine in Sport, 21(9), 935-940. https://doi.org/10.1016/j.jsams.2018.02.002

García-Hermoso, A., Ramírez-Campillo, R., \& Izquierdo, M. (2019). Is Muscular Fitness Associated with Future Health Benefits in Children and Adolescents? A Systematic 
Review and Meta-Analysis of Longitudinal Studies. Sports Medicine, 49(7), 1079-1094. https://doi.org/10.1007/s40279-019-01098-6

Hauspie, R. C., Vercauteren, M., \& Susanne, C. (1997). Secular changes in growth and maturation: An update. Acta Paediatrica, 86(S423), 20-27. https://doi.org/10.1111/j.1651-2227.1997.tb18364.x

Hunsicker, P., \& Reiff, G. (1977). Youth Fitness Report: 1958-1965-1975. Journal of Physical Education and Recreation, 48(1), 31-33. https://doi.org/10.1080/00971170.1977.10617562

Ignasiak, Z., Slawińska, T., \& Malina, R. M. (2016). Short term secular change in body size and physical fitness of youth 7-15 years in Southwestern Poland: 2001-2002 and 2010-2011. Anthropological Review, 79(3), 311-329.

Ikeda, N. (1962). A Comparison of Physical Fitness of Children in Iowa, U.S.A. and Tokyo, Japan. Research Quarterly. American Association for Health, Physical Education and Recreation, 33(4), 541-552. https://doi.org/10.1080/10671188.1962.10762105

Ingle, L., Sleap, M., \& Tolfrey, K. (2006). The effect of a complex training and detraining programme on selected strength and power variables in early pubertal boys. Journal of Sports Sciences, 24(9), 987-997. https://doi.org/10.1080/02640410500457117

Institute of Medicine. (2012). Fitness Measures and Health Outcomes in Youth. National Academies Press.

Karpati, A. M., Rubin, C. H., Kieszak, S. M., Marcus, M., \& Troiano, R. P. (2002). Stature and pubertal stage assessment in American boys: The 1988-1994 Third National Health and Nutrition Examination Survey. The Journal of Adolescent Health: Official Publication of 
the Society for Adolescent Medicine, 30(3), 205-212. https://doi.org/10.1016/s1054$139 x(01) 00320-2$

Katzmarzyk, P. T., Malina, R. M., \& Beunen, G. P. (1997). The contribution of biological maturation to the strength and motor fitness of children. Annals of Human Biology, 24(6), 493-505. https://doi.org/10.1080/03014469700005262

Katzmarzyk, P. T., \& Mason, C. (2009). The physical activity transition. Journal of Physical Activity \& Health, 6(3), 269-280. https://doi.org/10.1123/jpah.6.3.269

Koebel, C. I., Swank, A. M., \& Shelburne, L. (1992). Fitness Testing in Children: A Comparison Between PCPFS and AAHPERD Standards. The Journal of Strength \& Conditioning Research, 6(2), 107-114.

Lamoureux, N. R., Fitzgerald, J. S., Norton, K. I., Sabato, T., Tremblay, M. S., \& Tomkinson, G. R. (2019). Temporal Trends in the Cardiorespiratory Fitness of 2,525,827 Adults Between 1967 and 2016: A Systematic Review. Sports Medicine, 49(1), 41-55. https://doi.org/10.1007/s40279-018-1017-y

Leong, D. P., Teo, K. K., Rangarajan, S., Lopez-Jaramillo, P., Avezum, A., Orlandini, A., ... Yusuf, S. (2015). Prognostic value of grip strength: Findings from the Prospective Urban Rural Epidemiology (PURE) study. The Lancet, 386(9990), 266-273. https://doi.org/10.1016/S0140-6736(14)62000-6

Mainous, A. G., Tanner, R. J., Anton, S. D., \& Jo, A. (2015). Grip Strength as a Marker of Hypertension and Diabetes in Healthy Weight Adults. American Journal of Preventive Medicine, 49(6), 850-858. https://doi.org/10.1016/j.amepre.2015.05.025

Malina, R. M. (1978). Secular changes in growth, maturation, and physical performance. Exercise and Sport Sciences Reviews, 6, 203-255. 
Malina, R. M. (2004). Secular trends in growth, maturation and physical performance. A review. Anthropological Review, 67, 3-31.

McGrath, R., Robinson-Lane, S. G., Peterson, M. D., Bailey, R. R., \& Vincent, B. M. (2018). Muscle Strength and Functional Limitations: Preserving Function in Older Mexican Americans. Journal of the American Medical Directors Association, 19(5), 391-398. https://doi.org/10.1016/j.jamda.2017.12.011

Milliken, L. A., Faigenbaum, A. D., Loud, R. L., \& Westcott, W. L. (2008). Correlates of Upper and Lower Body Muscular Strength in Children. The Journal of Strength \& Conditioning Research, 22(4), 1339-1346. https://doi.org/10.1519/JSC.0b013e31817393b1

Moher, D., Liberati, A., Tetzlaff, J., \& Altman, D. G. (2009). Preferred reporting items for systematic reviews and meta-analyses: The PRISMA statement. BMJ, 339, b2535. https://doi.org/10.1136/bmj.b2535

Moliner-Urdiales, D., Ruiz, J. R., Ortega, F. B., Jiménez-Pavón, D., Vicente-Rodriguez, G., ReyLópez, J. P., ... Moreno, L. A. (2010). Secular trends in health-related physical fitness in Spanish adolescents: The AVENA and HELENA Studies. Journal of Science and Medicine in Sport, 13(6), 584-588. https://doi.org/10.1016/j.jsams.2010.03.004

Montpetit, R. R., Montoye, H. J., \& Laeding, L. (1967). Grip Strength of School Children, Saginaw, Michigan: 1899 and 1964. Research Quarterly. American Association for Health, Physical Education and Recreation, 38(2), 231-240. https://doi.org/10.1080/10671188.1967.10613384

Ogden, C. L., Flegal, K. M., Carroll, M. D., \& Johnson, C. L. (2002). Prevalence and trends in overweight among US children and adolescents, 1999-2000. JAMA, 288(14), 1728-1732. https://doi.org/10.1001/jama.288.14.1728 
Ortega, Francisco B., Ruiz, J. R., Castillo, M. J., \& Sjöström, M. (2008). Physical fitness in childhood and adolescence: A powerful marker of health. International Journal of Obesity, 32(1), 1-11. https://doi.org/10.1038/sj.ijo.0803774

Ortega, Francisco B., Silventoinen, K., Tynelius, P., \& Rasmussen, F. (2012). Muscular strength in male adolescents and premature death: Cohort study of one million participants. $B M J$, 345, e7279. https://doi.org/10.1136/bmj.e7279

Özen, S., \& Darcan, Ş. (2011). Effects of Environmental Endocrine Disruptors on Pubertal Development. Journal of Clinical Research in Pediatric Endocrinology, 3(1), 1-6. https://doi.org/10.4274/jcrpe.v3i1.01

Phillips, W. T., \& Haskell, W. L. (1995). "Muscular Fitness"-Easing the Burden of Disability for Elderly Adults. Journal of Aging and Physical Activity, 3(3), 261-289. https://doi.org/10.1123/japa.3.3.261

President's Council on Sports, Fitness \& Nutrition. (2018). Our History. U.S. Department of Health and Human Services.

Reiff, G., Dixon, W., Jacoby, D., Ye, X., Spain, C., \& Hunsiker, P. (1986). The President Council on Physical Fitness and Sports 1985. National School Population Survey. Washington, DC: President's Council of Physical Fitness and Sports.

Robbins, W. (1984). Alabama Motor Fitness and Health Related Fitness Norms for age 6-14 (Doctoral Dissertation). The University of Alabama, Tuscaloosa, United States.

Roche, A. F. (1979). Secular Trends in Stature, Weight, and Maturation. Monographs of the Society for Research in Child Development, 44(3/4), 3-27. https://doi.org/10.2307/1165883 
Rogol, A. D., Clark, P. A., \& Roemmich, J. N. (2000). Growth and pubertal development in children and adolescents: Effects of diet and physical activity. The American Journal of Clinical Nutrition, 72(2), 521S-528S. https://doi.org/10.1093/ajcn/72.2.521S

Ross, R., Blair, S. N., Arena, R., Church, T. S., Després, J.-P., Franklin Barry A., ... Wisløff Ulrik. (2016). Importance of Assessing Cardiorespiratory Fitness in Clinical Practice: A Case for Fitness as a Clinical Vital Sign: A Scientific Statement From the American Heart Association. Circulation, 134(24), e653-e699.

https://doi.org/10.1161/CIR.0000000000000461

Ruiz, J. R., Castro-Piñero, J., España-Romero, V., Artero, E. G., Ortega, F. B., Cuenca, M. M., ... Castillo, M. J. (2011). Field-based fitness assessment in young people: The ALPHA health-related fitness test battery for children and adolescents. British Journal of Sports Medicine, 45(6), 518-524. https://doi.org/10.1136/bjsm.2010.075341

Sandercock, G. R. H., \& Cohen, D. D. (2019). Temporal trends in muscular fitness of English 10-year-olds 1998-2014: An allometric approach. Journal of Science and Medicine in Sport, 22(2), 201-205. https://doi.org/10.1016/j.jsams.2018.07.020

Silverman, I. W. (2011). The secular trend for grip strength in Canada and the United States. Journal of Sports Sciences, 29(6), 599-606. https://doi.org/10.1080/02640414.2010.547209

Silverman, I. W. (2015). Age as a moderator of the secular trend for grip strength in Canada and the United States. Annals of Human Biology, 42(3), 201-211. https://doi.org/10.3109/03014460.2014.934920

Smith, J. J., Eather, N., Morgan, P. J., Plotnikoff, R. C., Faigenbaum, A. D., \& Lubans, D. R. (2014). The Health Benefits of Muscular Fitness for Children and Adolescents: A 
Systematic Review and Meta-Analysis. Sports Medicine, 44(9), 1209-1223.

https://doi.org/10.1007/s40279-014-0196-4

Smith, J. J., Eather, N., Weaver, R. G., Riley, N., Beets, M. W., \& Lubans, D. R. (2019).

Behavioral Correlates of Muscular Fitness in Children and Adolescents: A Systematic Review. Sports Medicine, 49(6), 887-904. https://doi.org/10.1007/s40279-019-01089-7

Smpokos, E. A., Linardakis, M., Papadaki, A., Lionis, C., \& Kafatos, A. (2012). Secular trends in fitness, moderate-to-vigorous physical activity, and TV-viewing among first grade school children of Crete, Greece between 1992/93 and 2006/07. Journal of Science and Medicine in Sport, 15(2), 129-135. https://doi.org/10.1016/j.jsams.2011.08.006

Tomkinson, G. R., Carver, K. D., Atkinson, F., Daniell, N. D., Lewis, L. K., Fitzgerald, J. S., ... Ortega, F. B. (2018). European normative values for physical fitness in children and adolescents aged 9-17 years: Results from 2779165 Eurofit performances representing 30 countries. British Journal of Sports Medicine, 52(22), 1445-1456. https://doi.org/10.1136/bjsports-2017-098253

Tomkinson, Grant R. (2007). Global changes in anaerobic fitness test performance of children and adolescents (1958-2003). Scandinavian Journal of Medicine \& Science in Sports, 17(5), 497-507. https://doi.org/10.1111/j.1600-0838.2006.00569.x

Tomkinson, Grant R., Lang, J. J., \& Tremblay, M. S. (2019). Temporal trends in the cardiorespiratory fitness of children and adolescents representing 19 high-income and upper middle-income countries between 1981 and 2014. British Journal of Sports Medicine, 53(8), 478-486. https://doi.org/10.1136/bjsports-2017-097982

Venckunas, T., Emeljanovas, A., Mieziene, B., \& Volbekiene, V. (2017). Secular trends in physical fitness and body size in Lithuanian children and adolescents between 1992 and 
2012. Journal of Epidemiology and Community Health, 71(2), 181-187.

https://doi.org/10.1136/jech-2016-207307

Weber, J. C. (1969). The Physical Fitness Status of Oklahoma Youth. (A Pilot Study for Grades 7 and 8). Final Report. Retrieved from https://eric.ed.gov/?id=ED035061

Weinberg, R., Tenenbaum, G., McKenzie, A., Jackson, S., Anshel, M., Grove, R., \& Fogarty, G. (2000). Motivation for youth participation in sport and physical activity: Relationships to culture, self-reported activity levels, and gender. International Journal of Sport Psychology, 31(3), 321-346.

Welk, G. J., Bai, Y., Saint-Maurice, P. F., Allums-Featherston, K., \& Candelaria, N. (2016). Design and Evaluation of the NFL PLAY 60 FITNESSGRAM Partnership Project. Research Quarterly for Exercise and Sport, 87(1), 1-13. https://doi.org/10.1080/02701367.2015.1127126 